\title{
$5-2018$
}

\section{Cardiovascular disease prevention: Training opportunities, the challenges, and future directions}

\author{
Anum Saeed \\ Baylor College of Medicine, United States \\ Kaustubh Dabhadkar \\ Alpert Medical School of Brown University, United States \\ Salim S. Virani \\ Aga Khan University, salim.virani@aku.edu \\ Peter H. Jones \\ Baylor College of Medicine, United States \\ Christie M. Ballantyne \\ Baylor College of Medicine, United States
}

See next page for additional authors

Follow this and additional works at: https://ecommons.aku.edu/pakistan_fhs_mc_med_cardiol

Part of the Cardiology Commons, and the Cardiovascular Diseases Commons

\section{Recommended Citation}

Saeed, A., Dabhadkar, K., Virani, S. S., Jones, P. H., Ballantyne, C. M., Nambi, V. (2018). Cardiovascular disease prevention: Training opportunities, the challenges, and future directions. Current Atherosclerosis Reports, 20(7), 35.

Available at: https://ecommons.aku.edu/pakistan_fhs_mc_med_cardiol/53 


\section{Authors}

Anum Saeed, Kaustubh Dabhadkar, Salim S. Virani, Peter H. Jones, Christie M. Ballantyne, and Vijay Nambi 


\title{
Cardiovascular Disease Prevention: Training Opportunities, the Challenges, and Future Directions
}

\author{
Anum Saeed $^{1,2} \cdot$ Kaustubh Dabhadkar $^{3} \cdot$ Salim S. Virani ${ }^{1,2,4,5,6} \cdot$ Peter H. Jones $^{2} \cdot$ Christie M. Ballantyne $^{1,2,7}$. \\ Vijay Nambi ${ }^{1,2,4,8}$
}

Published online: 21 May 2018

(C) This is a U.S. Government work and not under copyright protection in the US; foreign copyright protection may apply 2018

\begin{abstract}
Purpose Cardiovascular diseases (CVDs) are a leading cause of morbidity and mortality worldwide, necessitating major efforts in prevention. This review summarizes the currently available training opportunities in CVD prevention for fellows-in-training (FITs) and residents. We also highlight the challenges and future directions for CVD prevention as a field and propose a structure for an inclusive CVD prevention training program.

Recent Findings At present, there is a lack of centralized training resources for FITs and residents interested in pursuing a career in CVD prevention. Training in CVD prevention is not an accredited subspecialty fellowship by the American Council of Graduate Medical Education (ACGME). Although there are several independent training programs under the broad umbrella of CVD prevention focusing on different aspects of prevention, there is no unified curriculum or training.

Summary More collaborative efforts are needed to identify CVD prevention as an ACGME-accredited subspecialty fellowship. Providing more resources can encourage and produce more leaders in this essential field.
\end{abstract}

Keywords Heart disease prevention · CVD prevention training · Cardiology training programs · Preventive cardiology training · Fellowship $\cdot$ Fellows-in-training

This article is part of the Topical Collection on Coronary Heart Disease

Vijay Nambi

vnambi@bcm.edu

1 Section of Cardiovascular Research, Department of Medicine, Baylor College of Medicine, Houston, TX, USA

2 Center for Cardiometabolic Disease Prevention, Baylor College of Medicine, Houston, TX, USA

3 Division of Cardiology, Department of Medicine, Alpert Medical School of Brown University, Providence, RI, USA

4 Section of Cardiology, Michael E. DeBakey Veterans Affairs Medical Center, Houston, TX, USA

5 Health Policy, Quality and Informatics Program, Michael E. DeBakey Veterans Affairs Medical Center Health Services Research and Development Center for Innovations, Houston, TX, USA

6 The Aga Khan University, Karachi, Pakistan

7 Section of Cardiology, Department of Medicine, Baylor College of Medicine, Houston, TX, USA

86655 Travis Street, Suite 320, Houston, TX 77030, USA

\section{Introduction}

Approximately 2200 Americans die from cardiovascular disease (CVD)-related causes each day, an average of 1 death every $40 \mathrm{~s}$ [1]. According to the 2017 heart disease and stroke statistics update by the American Heart Association (AHA), about 92.1 million American adults are living with some form of CVD including coronary heart diseases (CHDs) or strokes. There is also an alarming increase in the incidence of CHD among younger adults [1]. The annual direct and indirect costs from CHD and stroke is estimated to total more than \$316 billion when including both health expenditures and lost productivity.

There is robust evidence that CVD is a potentially modifiable disease. Although atherosclerosis is a life-long process, risk factor modification helps with the prevention of CVD events and associated morbidities. Additionally, with the ever-growing need for cost-efficient delivery of care and introduction of quality payment programs, most notably the Medicare and CHIP Reauthorization (MACRA) Act 2015, the focus has shifted to high-quality care at a low cost. 
Preventive strategies have been shown to have the lowest cost per quality-adjusted life-years [2].

Data also suggest that many patients who are at high risk of CVD or those with established coronary diseases or strokes remain unidentified and/or inadequately treated [3]. Pokharel et al. have previously shown suboptimal statin use and facility-level variation in statin prescriptions for patients at high risk for CHD [3]. Results from the PINNACLE (Practice INNovation And CLinical Excellence) registry, a national cardiology outpatient quality improvement registry which extracts data from electronic medical records, revealed that approximately $38 \%$ of patients with diabetes in cardiology practices had no documentation of a statin prescription [4]. We know that individuals with familial hypercholesterolemia $(\mathrm{FH})$, if untreated, are at least 20 times more likely to have incident CHD events than those without FH [5]. Early crosssectional data from the national FH registry (CASCADE$\mathrm{FH})$ showed that the median age of diagnosis for adults with FH in the USA was 47 years [3]. Currently, not only are individuals with FH likely to be diagnosed very late in life but also under-treated, with only $25 \%$ patients in the CASCADE-FH registry reaching low-density lipoprotein cholesterol levels $<100 \mathrm{mg} / \mathrm{dL}$ [3]. A recent study showed subclinical atherosclerosis can be detected by ultrasonography and coronary calcium tests in a cohort of middleaged individuals without traditional risk factors for CVD [6]; highlighting the potential need for early risk stratification [7]. Education in CVD prevention including familiarity and use of newer diagnostic modalities are key to a more comprehensive detection of high-risk CVD populations. Newer biomarkers such as high-sensitivity troponin $\mathrm{T}$ and $\mathrm{N}$-terminal pro brain natriuretic peptide (NTproBNP) measured by very sensitive assays can diagnose subclinical myocardial injury, and these biomarkers have been shown to improve risk prediction of coronary heart diseases and heart failure. Use of coronary artery calcium scoring has been established as a diagnostic marker for subclinical atherosclerosis. Thus, a number of diagnostic modalities have a potential to be highly useful in clinical practices for CVD prevention.

Cardiologists and primary care providers have a crucial role to play in prevention of CVD events; by first and foremost, identifying high-risk individuals, then applying lifestyle interventions and pharmacotherapies, and coordinating multidisciplinary care, including cardiac rehabilitation, when indicated. However, most of the experience in this field comes "on the fly" while training. Currently, there is a lack of strategically designed subspecialty training in CVD prevention. Further, there is an absence of consensus on the necessary curriculum for training programs as well as a lack of a centralized resources for CVD prevention training programs $[8 \cdot \bullet]$.
In this article, we review available resources for CVD prevention training. Further, we highlight the leading challenges for training in this niche area of CVD prevention.

\section{Status of Cardiovascular Disease Prevention Training in the USA}

Over the last two decades, there has been incremental growth in our understanding of CVD risk factors. Hence, there has been a rapid influx of newer guidelines related to lipids, hypertension, diabetes mellitus, obesity, thrombosis, and vascular diseases. As a result, various independently funded fellowships have emerged with a focus on these specific disciplines, aimed at providing advanced training.

In 2012, a survey of the US cardiology fellowship training programs revealed that only $24 \%$ of the surveyed programs met the Core Cardiovascular Training Symposium (COCATS) guidelines with a dedicated 1-month rotation in preventive cardiology (used interchangeably with CVD prevention). Approximately, 24\% of the programs had no formalized training in CVD prevention and 30\% had no faculty with expertise in the field [9]. "Lack of time" and "lack of a developed curriculum in prevention" were cited as the most prominent barriers to training in CVD prevention by program directors and chief fellows of the surveyed programs [9].

Following this survey, the COCATS 4 Task Force 2 guidelines were published in 2015 and endorsed by the American board of Internal Medicine (ABIM) and Accreditation Council of Graduate Medical Education (ACGME) [3]. This document recognized primary and secondary atherosclerotic CVD prevention strategies as core learning principles during general cardiology fellowship training. As with most other adult CVD areas, three levels of training were described and are currently applicable $[10 \bullet \bullet]$, as follows:

- Level 1: Basic training required for all physicians to become competent cardiologists. This level of training can be achieved by at least 1 month of training dedicated to CVD prevention medicine. This 1-month rotation should encompass weekly attendance at a cardiac rehabilitation program, diabetes or endocrinology clinic, hypertension clinic, and dyslipidemia management clinic.

An alternative to the 1 month dedicated to CVD prevention is a 3-month (or longer) training in comprehensive cardiac rehabilitation with at least weekly attendance.

- Level 2: Additional training in "1 or more areas that enable cardiologists to perform or interpret specific procedures or render more specialized care for specific patients and conditions." For prevention, the Task Force identified no specific competencies for Level II training. 
- Level 3: For trainees who are interested in seeking expertise to attain program director-level positions in clinical, research or combined programs. No formal level III training program or curriculum has been identified for CVD prevention as of yet. Trainees are encouraged to attain additional (currently non ACGME-accredited) fellowships to achieve level III.

The core competencies in CVD prevention modules (graded by milestones) for trainees were also delineated by the COCATS 4 Task Force guidelines which included six domains (medical knowledge, patient care and procedural skills, system-based practice, practice-based learning and improvement, professionalism and interpersonal and communication skills). Further, a strong emphasis was placed on learning risk factor modification strategies in curtailing the progression of subclinical atherosclerosis as well as CHD-associated morbidity and mortality $[10 \bullet \cdot]$.

As a result, general cardiology fellow in training (FITs) are mandated to spend at least 1 month of their 36-month training period in a prevention setting to obtain the minimum level of expertise in CVD prevention and meet the aforementioned milestones. Although COCATs level I preventive training is required of all cardiology FITs [10••], trainees who are interested in pursuing a career in CVD prevention will likely require an additional $1-2$ years of training.

\section{Potential Challenges in CVD Prevention Training}

The initial challenge in pursuing a career in CVD prevention is the absence of centralized resources or consolidated catalog of prospective training opportunities for fellows or residents. Similarly, there is no central board/society which would allow certification in preventive cardiology. Furthermore, candidates who are on a visa may face additional difficulty as their visas may not be acceptable for training at many of the programs.

Unlike the traditional medicine residencies or ABIM recognized subspecialty fellowships, CVD prevention fellowships do not have an "Electronic Residency Application Service" (ERAS) like application portal which streamlines the application process, includes a list of all currently available programs, and manages application submissions as well as communication related to interview invitations.

Although several societies provide guidelines and recommendations on management of various aspects of CVD prevention [11-13], there is currently a lack of a formalized curriculum for CVD prevention fellowship training which has been endorsed uniformly across all societies. Further, the duration of a CVD prevention fellowship may vary from institution to institution. Depending on the program, training may be 1 or 2 years and include research and/or clinical time to various degrees. Most 2-year fellowships include a research year with focuses including basic science, genetics, translational, and/or outcomes/quality improvement. Given the growing need for this subspecialty in the USA and worldwide, there have been recent suggestions for ABIM accreditation of preventive cardiology as a fifth subspecialty in cardiology $[8 \bullet \bullet]$.

\section{Current Programs and Available Certifications}

Various training options are available for interested candidates which may cover (but are not limited to) general topics in CVD prevention as below:

- Lipid and hypertension management strategies for primary and secondary prevention

- Obesity management

- Cardiac rehabilitation

- Cardiovascular genomics

- Design and conduct of clinical trials

- Biostatistics and epidemiological training in CVD

Pack et al. previously published a consolidated list of 15 such training programs [14]. However, given that this was published at least 5 years ago, changes in program websites, application deadlines, and training descriptions are likely. We have therefore outlined currently existing programs that we are aware of in Table 1. All programs were contacted to ensure ongoing training. Furthermore, current contact information is provided in this updated table.

In addition to these independently funded opportunities in CVD prevention training, there exists a comprehensive list of the National Heart Lung and Blood Institute (NHLBI)-funded Epidemiology and Statistical training programs for MDs and $\mathrm{PhDs}$ interested in CVD prevention on its official website [15]. These programs, which are primarily research-oriented and aimed towards training predoctoral and postdoctoral applicants, are an important pathway towards becoming an investigator in the field of CVD prevention. However, the NIH-funded programs are only open to US citizens or non-citizen nationals or green card holders and those on temporary or student visas are generally not eligible to apply for these positions.

We are not certain how many prospective applicants are familiar with the NHLBI website resources. Therefore, we have summarized some of these programs in Table 2. A complete list of the NHLBI-funded training programs can also be found at https://www.nhlbi.nih.gov/research/training/epi-bio.

Given that there is no ACGME accreditation for CVD prevention, the programs are in general sponsored by the local state medical board. The lack of ownership by a centralized board for CVD prevention training is a major limiting factor in formalized learning for this essential subspecialty.

Currently, the National Lipid Association independently controls the American Board of Clinical Lipidology, which provides a certification in lipidology which is not affiliated with or endorsed by the ABIM. It also provides an annual Masters in 

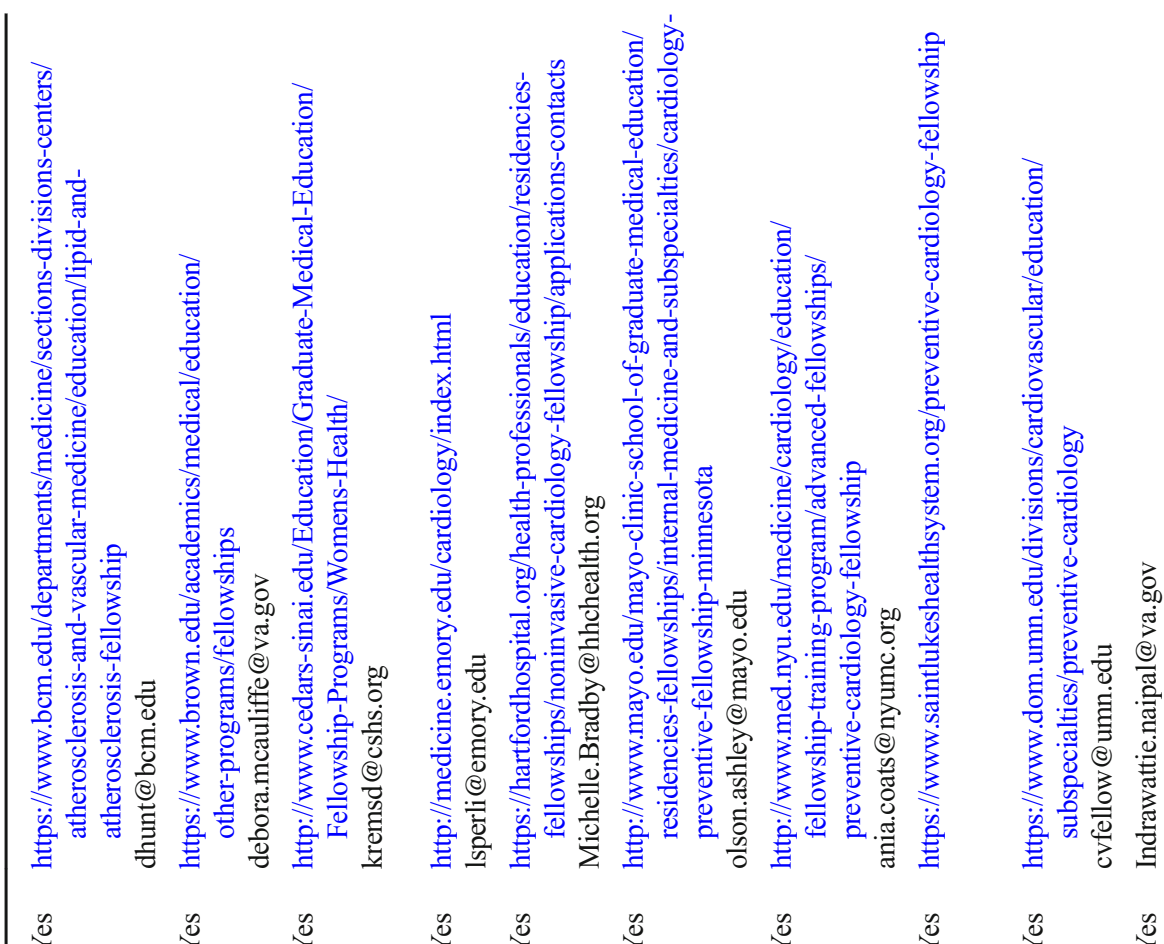

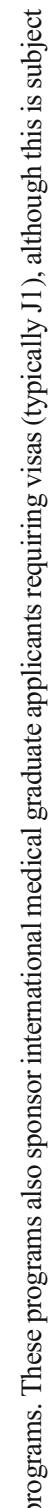

5

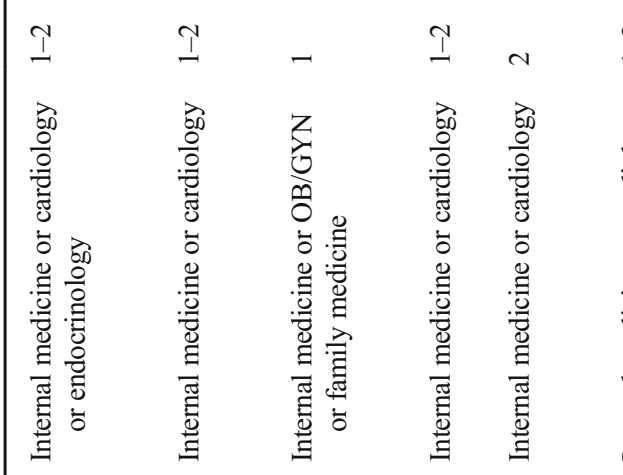

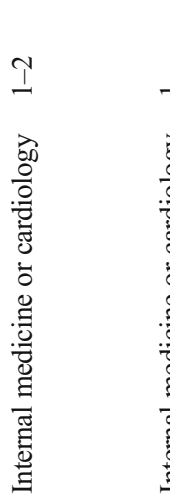

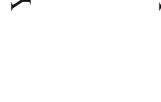

$\approx \quad 2 \quad 0$

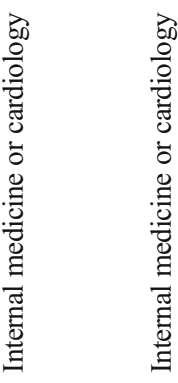

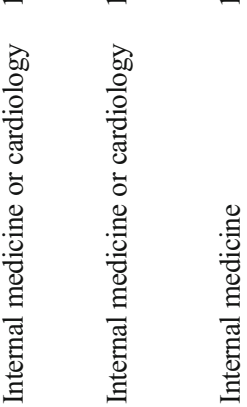

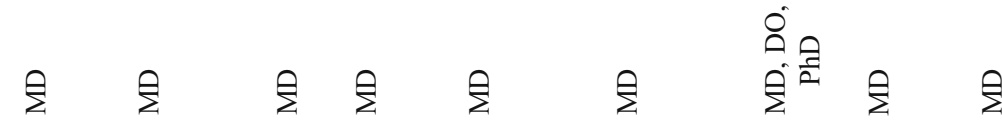

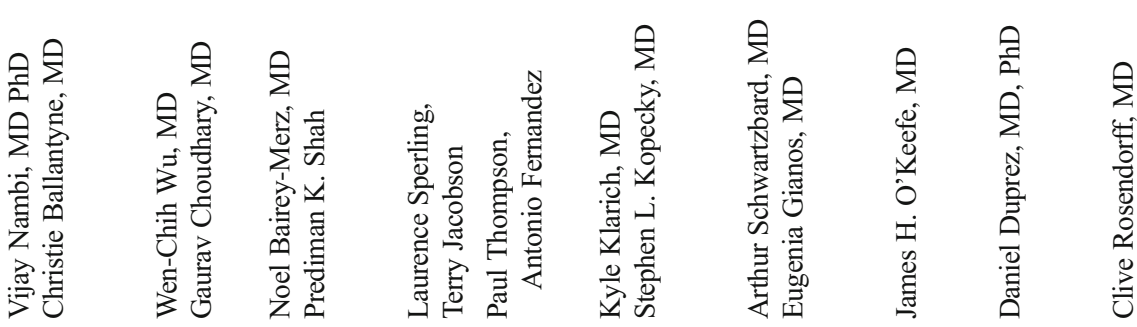

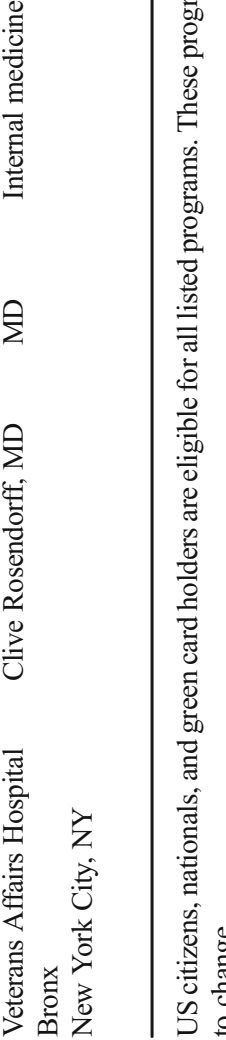

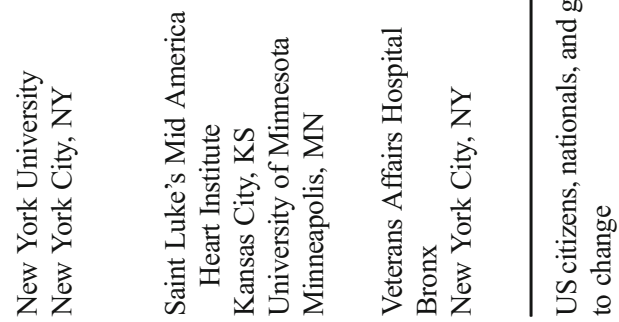




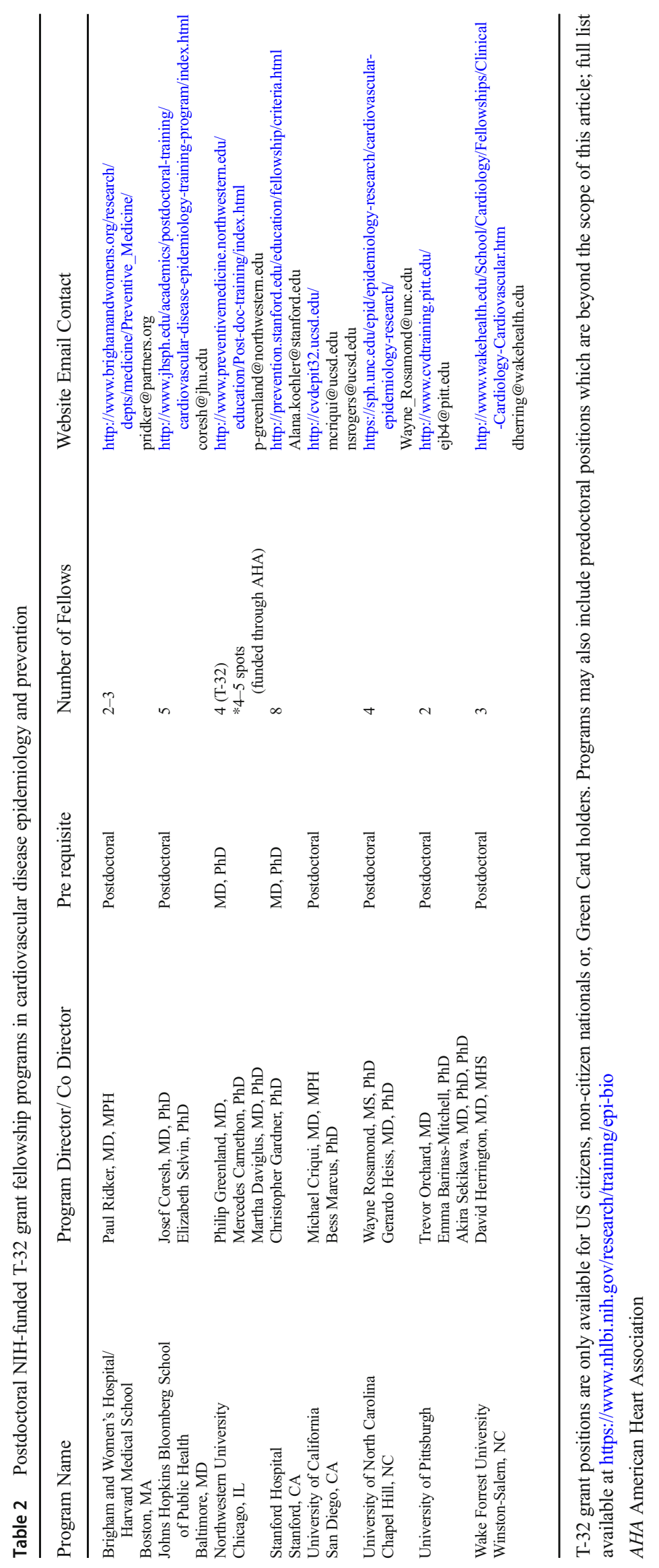


Clinical Lipidology course for interested trainees and other healthcare providers. Other societies which provide board certifications or online continuing medical education (CME) opportunities include The American Society of Preventive Cardiology (Online CME courses), American Society of Hypertension (Specialist in Clinical Hypertension Certification and Certified Hypertension Clinician Certificate), American Obesity Society (American Board of Obesity Management Certification), and American Association of Diabetes Educators (Board Certification in Advanced Diabetes Management). More information on these courses are detailed on the respective organization's official websites.

\section{Directions for the Future}

Prevention of CVD remains one of the major goals for the scientific and healthcare community. Therefore, CVD prevention (both primary and secondary) requires dedicated attention and training. However, preventive cardiology is a subspecialty that is at times, lost between clinical internal medicine, endocrinology, and cardiology with no clear champion. Resources and formalized structure in CVD prevention training are scant. Although the ACC COCATS guidelines provide a general framework and recommendations for basic training in $\mathrm{CV}$ prevention that is required of all training programs in cardiovascular medicine, several programs still lack the requirements for basic training in CVD prevention.

Moving forward, it is important for central educational bodies like the ABIM to recognize CVD prevention as a subspecialty of cardiovascular disease training. Leading professional organizations like the American College of Cardiology (ACC) and American Heart Association (AHA) can collaborate with other organizations focused on CVD prevention to provide resources and help develop CVD prevention training programs. There are several ways ACC/AHA can collaborate with the CVD training programs which include (and may not be limited to) creating a central resource list for training opportunities which is readily accessible for trainees and providing resources to institutions with CVD fellowship programs, such as offering online curriculum focused on preventative training (lipid disorder management, smoking cessation, resistant hypertension, and cardiac rehabilitation).

\section{Potential Structure of Fellowship Training}

Although the structure of currently available fellowships in CVD prevention depends on the specific home institution, moving forward, an ACGME-approved curriculum will be beneficial for comprehensive training. In our opinion, a fellowship program for CVD prevention should aim to train both clinicians and physician-scientists who will advance the field. Shapiro et al. have recently suggested what preventive cardiology fellowships should include (8). Table 3 illustrates our proposed structure for a CVD prevention fellowship training program.

The clinician track may encompass a 1-year clinical training where FITs train in lipid, diabetes, hypertension, obesity, and cardiac rehabilitation which will aid them with the management
Table 3 Suggested cardiovascular disease prevention training program outline

\begin{tabular}{|c|c|c|}
\hline Track & Clinical Track & Physician-Scientist Track \\
\hline Duration (years) & 1 & $1-2$ \\
\hline Clinical Exposure $^{\mathrm{a}}$ & 3 or more half day clinics per week & 1-2 half-day clinics per week \\
\hline \multicolumn{3}{|l|}{ - Lipid clinic } \\
\hline \multicolumn{3}{|l|}{ - Hypertension } \\
\hline \multicolumn{3}{|l|}{ - Diabetes management } \\
\hline \multicolumn{3}{|l|}{ - Cardiac rehabilitation } \\
\hline \multicolumn{3}{|c|}{ - Dietician/lifestyle management } \\
\hline \multicolumn{3}{|l|}{ - Heart failure $\mathrm{b}^{\mathrm{b}}$ Prevention } \\
\hline \multicolumn{3}{|l|}{$\begin{array}{l}\text { Adolescent preventive } \\
\text { medicine clinic }\end{array}$} \\
\hline \multirow[t]{4}{*}{ Research training } & $\begin{array}{l}\text { Basic research methodology and } \\
\text { statistics understanding }\end{array}$ & \\
\hline & $>1$ publication per year & $\begin{array}{l}\text { Atleast } 1 \text { project/publication } \\
\text { in the year }\end{array}$ \\
\hline & & Grant writing training \\
\hline & & $\begin{array}{l}\text { Additional; MPH/MSCR Course } \\
\text { Degree/CSTP Course }\end{array}$ \\
\hline Conferences/Journal Club & Weekly & Weekly \\
\hline
\end{tabular}

CSTP Clinician-Scientist Training Program, MPH Masters in Public Health, MSCR Masters in Science in Clinical Research

${ }^{\text {a }}$ Clinical exposure includes multiple clinics including but not limited to the mentioned clinical training themes

${ }^{\mathrm{b}}$ Heart Failure Prevention is, at present, not an established clinical model 
of complex cases referred by other specialists. For the physicianscientist track, training will be geared towards producing researchers in CVD prevention. The physician-scientist track FITs would benefit from clinical exposure during the course of their fellowship. However, more time would likely be allocated to learning research methodology and grant writing skills.

In the future, another aspect of a CVD prevention fellowship could be dedicated to primordial prevention in addition to primary and secondary prevention. Collaboration between adult and pediatric cardiologists could allow FITs to spend time acquiring skills necessary for evaluation of children/teenagers/adolescents who are at high risk for developing CVD and associated risk factors like diabetes and hypertension.

\section{Conclusions}

In today's era, knowledge of the various aspects of CVD prevention is becoming increasingly relevant. CVD prevention is fundamental to global cardiovascular care, and there is room for improvement in training cardiology FITs and other trainees interested in this field. The central educational bodies should take important further steps such as recognizing CVD prevention as a subspecialty of cardiovascular medicine as well as creating and endorsing a central portal for the training opportunities in this field. These measures can aid trainees in furthering their clinical and research expertise in this area and provide networking opportunities with faculty and other trainees with a focus in CVD prevention. The ACC/AHA can significantly help further this mission. It is our belief and hope that implementation of such measures will encourage and prepare more leaders in the field of CVD prevention.

\section{Compliance with Ethics Guidelines}

Conflict of Interest Anum Saeed and Kaustubh Dabhadkar declare no conflict of interest. Salim S. Virani receives an honorarium from the American College of Cardiology as Associate Editor for Innovations for ACC.org. Peter H. Jones has been a scientific consultant to Amgen and to Sanofi/Regeneron the past 12 months. Vijay Nambi was on a regional advisory board for Sanofi and is named on provisional patent no. 61721475 entitled "Biomarkers to Improve Prediction of Heart Failure Risk" filed by Baylor College of Medicine and Roche. Christie M. Ballantyne has received grant/research support (all paid to institution, not individual) from Abbott Diagnostic, Amarin, Amgen, Eli Lilly, Esperion, Ionis, Novartis, Pfizer, Regeneron, Roche Diagnostic, SanofiSynthelabo, NIH, AHA, and ADA; has been a consultant for Abbott Diagnostics, Amarin, Amgen, Astra Zeneca, Boehringer Ingelheim, Eli Lilly, Esperion, Ionis, Matinas BioPharma Inc., Merck, Novartis, Pfizer, Regeneron, Roche Diagnostic, and Sanofi-Synthelabo; and is named on provisional patent no. 61721475 entitled "Biomarkers to Improve Prediction of Heart Failure Risk" filed by Baylor College of Medicine and Roche.

Human and Animal Rights and Informed Consent This article does not contain any studies with human or animal subjects performed by any of the authors.

\section{References}

Papers of particular interest, published recently, have been highlighted as:

- Of major importance

1. Benjamin EJ, Blaha MJ, Chiuve SE, Cushman M, Das SR, Deo R, et al. Heart disease and stroke Statistics-2017 update: a report from the American Heart Association. Circulation. 2017;135:e146-603.

2. Cohen JT, Neumann PJ, Weinstein MC. Does preventive care save money? Health economics and the presidential candidates. N Engl J Med. 2008;358:661-3.

3. Nambi V, Liu X, Chambless LE, de Lemos JA, Virani SS, Agarwal $\mathrm{S}$, et al. Troponin T and $\mathrm{N}$-terminal pro-B-type natriuretic peptide: a biomarker approach to predict heart failure risk - the atherosclerosis risk in communities study. Clin Chem. 2013;59:1802-10.

4. Pokharel Y, Gosch K, Nambi V, Chan PS, Kosiborod M, Oetgen WJ, et al. Practice-level variation in statin use among patients with diabetes: insights from the PINNACLE registry. J Am Coll Cardiol. 2016;68:1368-9.

5. Onorato A, Sturm AC. Heterozygous familial hypercholesterolemia. Circulation. 2016;133:e587-9.

6. Fernandez-Friera L, Fuster V, Lopez-Melgar B, et al. Normal LDLcholesterol levels are associated with subclinical atherosclerosis in the absence of risk factors. J Am Coll Cardiol. 2017;70:2979-91.

7. Nambi V, Bhatt DL. Primary prevention of atherosclerosis: time to take a selfie? J Am Coll Cardiol. 2017;70:2992-4.

8.• Shapiro MD, Fazio S. Setting the agenda for preventive cardiology. Circ Res. 2017;121:211-3. A comprehensive paper which described an potential preventive cardiology service for practical purposes and preventive cardiology training outline.

9. Pack QR, Keteyian SJ, McBride PE, Weaver WD, Kim HE. Current status of preventive cardiology training among United States cardiology fellowships and comparison to training guidelines. Am J Cardiol. 2012;110:124-8.

10.• Smith SC Jr, Bittner V, Gaziano JM, et al. COCATS 4 task force 2: training in preventive cardiovascular medicine. J Am Coll Cardiol. 2015;65:1754-62. An important document published by the American College of Cardiology on the requirements for trainees for achieving milestones in cardiovascular disease prevention during their general cardiovascular disease fellowship training.

11. Jacobson TA, Ito MK, Maki KC, Orringer CE, Bays HE, Jones PH, et al. National lipid association recommendations for patientcentered management of dyslipidemia: part 1-full report. J Clin Lipidol. 2015;9:129-69.

12. Lloyd-Jones DM, Morris PB, Ballantyne CM, et al. ACC expert consensus decision pathway on the role of non-statin therapies for LDL-cholesterol lowering in the management of atherosclerotic cardiovascular disease risk: a report of the American College of Cardiology Task Force on clinical expert consensus documents. J Am Coll Cardiol. 2016;68:92-125.

13. Jellinger PS, Handelsman Y, Rosenblit PD, Bloomgarden ZT, Fonseca VA, Garber AJ, et al. American Association of Clinical Endocrinologists and American College of Endocrinology guidelines for management of dyslipidemia and prevention of cardiovascular disease. Endocr Pract. 2017;23:1-87.

14. Pack QR, Keteyian SJ, McBride PE. Subspecialty training in preventive cardiology: the current status and discoverable fellowship programs. Clin Cardiol. 2012;35:286-90.

15. NHLBI. National Heart Lung and blood institute, National Research Service Award Programs in cardiovascular epidemiology, biostatistics, and Prev Med 2017. 